\title{
Recent Discoveries in Monogenic Disorders of Childhood Bone Fragility
}

\section{Makitie, Riikka E.}

2017-08

Makitie , R E , Kampe , A J , Taylan , F \& Makitie , O 2017 , ' Recent Discoveries in Monogenic Disorders of Childhood Bone Fragility ' , Current Osteoporosis Reports (Online), vol. 15 , no. 4 , pp. 303-310 . https://doi.org/10.1007/s11914-017-0388-6

http://hdl.handle.net/10138/241195

https://doi.org/10.1007/s11914-017-0388-6

unspecified

publishedVersion

Downloaded from Helda, University of Helsinki institutional repository.

This is an electronic reprint of the original article.

This reprint may differ from the original in pagination and typographic detail.

Please cite the original version. 


\title{
Recent Discoveries in Monogenic Disorders of Childhood Bone Fragility
}

\author{
Riikka E. Mäkitie ${ }^{1}$ • Anders J. Kämpe ${ }^{2}$ - Fulya Taylan ${ }^{2}$ • Outi Mäkitie 1,2,3,4
}

Published online: 23 June 2017

(C) Springer Science+Business Media, LLC 2017

\begin{abstract}
Purpose of Review This review summarizes our current knowledge on primary osteoporosis in children with focus on recent genetic findings.

Recent Findings Advances in genetic research, particularly next-generation sequencing, have found several genetic loci that associate with monogenic forms of inherited osteoporosis, widening the scope of primary osteoporosis beyond classical osteogenesis imperfecta. New forms of primary osteoporosis, such as those related to WNT1, PLS3, and XYLT2, have identified defects outside the extracellular matrix components and collagen-related pathways, in intracellular cascades directly affecting bone cell function.

Summary Primary osteoporosis can lead to severe skeletal morbidity, including abnormal longitudinal growth, compromised bone mass gain, and noticeable fracture tendency beginning at childhood. Early diagnosis and timely care are warranted to ensure the best achievable bone health. Future research will most likely broaden the spectrum of primary osteoporosis, hopefully provide more insight into the genetics governing bone health, and offer new targets for treatment.
\end{abstract}

This article is part of the Topical Collection on Pediatrics

Outi Mäkitie

outi.makitie@helsinki.fi

1 Folkhälsan Institute of Genetics, University of Helsinki, P. O. Box 63, FIN-00014 Helsinki, Finland

2 Department of Molecular Medicine and Surgery, Center for Molecular Medicine, Karolinska Institutet, Stockholm, Sweden

3 Department of Clinical Genetics, Karolinska University Hospital, Stockholm, Sweden

4 Children's Hospital, University of Helsinki and Helsinki University Hospital, Helsinki, Finland
Keywords Primary osteoporosis · WNT1 · PLS3 - XYLT2 · Osteogenesis imperfect

\section{Bone Health in Childhood}

The bone is an ever-evolving tissue that grows, remodels, and renews all throughout life. During childhood, bone production exceeds resorption and the highest amount of bone mass, termed "peak bone mass", is acquired by early adulthood. Later in life, bone resorption surpasses formation and bone mass slowly decreases. Thus, childhood is a pivotal time for bone health, and any factor that decreases bone formation, i.e., chronic illnesses, long-term medications, malnutrition, and endocrine disorders, diminishes the attained peak bone mass and predisposes to an increased risk for osteoporosis [1].

\section{Definition of Osteoporosis}

The bone is created to be both sturdy to stand individual's weight and flexible to enable one's movement. The most common skeletal disorder is osteoporosis, which refers to the weak bone that is depleted in sufficient bone mineral, resulting in a propensity to fracture. Osteoporosis leads to substantial morbidity and mortality yearly, causes significant human suffering, and is a high burden on health care. In adults, osteoporosis is defined by BMD, which is a measure of both the extracellular bone matrix and the amount of bone mineral, and is usually measured using dual-energy $\mathrm{X}$-ray absorptiometry (DXA). BMD measures correlate with fracture risk, and DXA $T$-score of or below -2.5 is considered diagnostic for osteoporosis in adults [2].

Diagnosis of pediatric osteoporosis, on the other hand, requires more than a low DXA-determined BMD. As growth 
and pubertal timing vary individually, one needs to consider age, sex, and body size-adjusted DXA values together with fracture history; deviations from normal BMD may be regarded pathologic only when present together with skeletal fragility. A pathologic fracture history is defined according to international guidelines as (i) one or more vertebral compression fractures in the absence of high-energy trauma, $a \geq 20 \%$ loss in vertebral anterior, middle, or posterior height is required for the diagnosis of compression fracture; (ii) $\geq 2$ clinically significant long bone fractures by age 10 years; or (iii) $\geq 3$ clinically significant long bone fractures by 16 years. On the other hand, presence of one or more vertebral compression fracture(s) suffice alone for the diagnosis of osteoporosis even in the presence of normal BMD $[1,3,4]$.

\section{Genetic Factors Determining Bone Health}

While BMD is greatly influenced by lifestyle factors, genetics are thought to be of key importance in governing and determining an individual's bone health. Bone mass is known to be a heritable trait, and several twin studies have confirmed genetic factors explaining up to $80 \%$ of variance in skeletal characteristics, i.e., microarchitectural properties, biochemistry, geometric anatomy, and bone mass accrual and loss. The genetics of bone health appear to be quite complex and while several approaches, such as family linkage and animal studies, candidate gene association studies, and genome-wide association studies (GWAS) have been successful, most of the specific genetic variants regulating our susceptibility to osteoporosis remain unknown [5-8].

Osteoporosis is commonly regarded as a polygenic trait, and several genes, each with a modest effect, determine the final BMD and fracture risk. Only rarely is osteoporosis monogenic, i.e., single gene mutations overrule the effects of other genetic and environmental factors and lead to skeletal fragility. In childhood-onset primary osteoporosis, skeletal changes are often recognized in early life and depending on their severity causing severe growth retardation, low-energy fractures, and skeletal deformities at a young age. While resulting skeletal features are predominantly due to the mutated causative gene, other polygenic and environmental factors may account for broad phenotypic variability even within a family.

\section{Classical OI}

Osteogenesis imperfecta (OI) is a heterogeneous group of connective tissue disorders and serves as the prime example of monogenic, heritable bone disorders affecting children. To date, mutations in 18 different genes have been identified as causative of OI, including autosomal dominant and recessive as well as X-chromosomal inheritance patterns. The resulting phenotypes vary in severity and clinical characteristics; according to the most recent skeletal dysplasia nosology [9•], different genetic forms of OI are subdivided into five types based on the degree of clinical severity rather than giving each genetic form a separate name $[9 \bullet, 10,11]$. In addition to severe osteoporosis and abnormal skeletal anatomy, OI patients' clinical phenotype is dominated by a variety of extra-skeletal clinical characteristics, such as compromised longitudinal growth, characteristic facial features, dental anomalies, joint hypermobility, blue sclerae, and impaired hearing $[9 \cdot, 10]$.

Differences in phenotypes arise depending on the diseasecausing gene (Table 1). Over $90 \%$ of OI cases result from molecular changes in the type I collagen-coding genes COL1A1 and COL1A2, which hamper the qualitative and quantitative production of the main extracellular bone matrix protein, type I collagen. In consequence, the microarchitecture of the bone is inappropriately organized and abnormally mineralized, leading to compromised bone strength-hence named the "brittle bones" disease. In general, milder forms of OI are caused by haploinsufficiency due to premature termination of the coding sequence. Thus, the encoded type I collagen is structurally normal but produced in inadequate amounts, leading to a mild phenotype. The more severe forms (Types 2-4), on the other hand, result from sequence variants that do not hamper the amount of protein produced but distort the helical structure of collagen. The resulting phenotypes range in severity from perinatal lethal (type 2) to moderate (type 4) or severe (type 3) OI. Several studies have further evaluated the relationship between the types of mutations with other clinical manifestations; BMD $Z$-scores and height vary according to the OI type, vertebral compression fractures are more common in helical mutations in $\alpha 1$ - than $\alpha 2$-chain, dentinogenesis imperfecta seems to be absent in OI caused by N-terminal helical mutations, and blue sclerae are linked to mutations in the $\alpha 1$-chain $[13,14]$.

Besides the COL1-genes, other OI causative genes most often alter the posttranslational modification of the type I collagen protein and include such genes as IFITM5, SERPINF1, LEPRE1, and CRTAP. These forms may mimic the classical OI phenotype but also include various other clinical characteristics that differ in skeletal and extra-skeletal manifestations, phenotype severity, and inheritance pattern $[9 \cdot, 10]$.

Recent research has brought light to various other genes affecting bone mass and shown the spectrum of primary osteoporosis to be much wider. These new genes cause skeletal defects independent of collagen production and most often affect osteoblast or osteoclast function directly. Mutations in the LDL receptor-related protein 5 (LRP5) gene are known to cause the autosomal recessive osteoporosis-pseudoglioma syndrome (OPPG) while heterozygous gain-of-function mutations in the same gene result in a high bone mass phenotype $[15,16]$. Also, mutations in the SOST gene alter function of sclerostin and lead to increased WNT signaling and high bone mass $[17,18]$. The three most recently characterized forms of 
Table 1 Osteogenesis imperfecta (OI) causing genes according to 2015 Nosology and Lindert et al. $[9 \cdot, 12]$

\begin{tabular}{|c|c|c|c|c|c|c|}
\hline \multirow[t]{2}{*}{ Gene } & \multirow[t]{2}{*}{ Protein } & \multicolumn{5}{|c|}{ OI Phenotype } \\
\hline & & 1 & 2 & 3 & 4 & 5 \\
\hline COL1A1 & Collagen type $1 \alpha-1$ chain & $\mathrm{x}$ & $\mathrm{x}$ & $\mathrm{x}$ & $\mathrm{x}$ & \\
\hline COL1A2 & Collagen type $1 \alpha-1$ chain & $\mathrm{x}$ & $\mathrm{x}$ & $\mathrm{x}$ & $\mathrm{x}$ & \\
\hline CRTAP & Cartilage-associated protein & & $\mathrm{x}$ & $\mathrm{x}$ & $\mathrm{x}$ & \\
\hline LEPRE1 & Prolyl 3-hydroxylase 1 & & $\mathrm{x}$ & $\mathrm{x}$ & & \\
\hline PPIB & Cyclophilin B & & $\mathrm{x}$ & $\mathrm{x}$ & $\mathrm{x}$ & \\
\hline SERPINH1 & Heat shock protein 47 & & & $\mathrm{x}$ & & \\
\hline BMP1 & Bone morphogenic protein 1 & & & $\mathrm{x}$ & & \\
\hline FKBP10 & Peptidyl-prolyl cis-trans isomerase & & & $\mathrm{x}$ & $\mathrm{x}$ & \\
\hline PLOD2 & Procollagen-lysine, 2-oxoglutarate & & & $\mathrm{x}$ & & \\
\hline SERPINF1 & Pigment-epithelium-derived factor & & & $\mathrm{x}$ & $\mathrm{x}$ & \\
\hline SP7 & Osterix & & & $\mathrm{x}$ & $\mathrm{x}$ & \\
\hline $\mathrm{WNT}^{\mathrm{a}}$ & Wingless-type MMTV integration site family, AR member 1 & & & $\mathrm{x}$ & $\mathrm{x}$ & \\
\hline TMEM38B & Trimeric intracellular cation channel B & & & $\mathrm{x}$ & & \\
\hline CREB3L1 & cAMP responsive element binding protein 3 -like 1 & & & $\mathrm{x}$ & & \\
\hline SEC24D & SEC24-related gene family, member D & & & $\mathrm{x}$ & & \\
\hline IFTM5 & Interferon-induced transmembrane protein 5 & & & & & $\mathrm{x}$ \\
\hline SPARC & Secreted protein, acidic and rich in cysteine & & & & $\mathrm{x}$ & \\
\hline MBTPS2 & Membrane bound transcription factor peptidase, site 2 & & & $\mathrm{x}$ & $\mathrm{x}$ & \\
\hline
\end{tabular}

OI phenotypes: Type 1: non-deforming OI with blue sclerae; Type 2: perinatally lethal OI; Type 3: progressively deforming; Type 4: common variable OI with normal sclerae; Type 5: OI with calcification in interosseous membranes

${ }^{a}$ Biallelic mutations cause OI, heterozygous mutations cause early-onset osteoporosis monogenic osteoporosis are WNT1, PLS3, and XYLT2, to be described in more detail below [19].

\section{WNT1 Osteoporosis}

WNT-proteins, a 19-member family of glycoproteins, have a broad role in various developmental and regenerative processes in numerous tissues. In the bone, WNTs affect all aspects of bone health from fetal skeletal development to bone mass accrual in childhood and maintenance of bone homeostasis in adulthood. WNTs signal through the WNT- $\beta$-cateninsignaling pathway by binding to a transmembrane dual receptor-complex composed of low-density lipoprotein receptor-related proteins (LRP5 or LRP6) and a seven transmembrane G-protein Frizzled (FZD). Activating this pathway inhibits proteosomal degradation, allowing subsequent nuclear translocation of accumulating $\beta$-catenin, which in turn leads to target gene expression and ultimately increased bone formation and decreased bone resorption [20••].

The role of the WNT- $\beta$-catenin-signaling pathway in skeletal pathology was first recognized through its key constituent proteins LRP5 and sclerostin. LRP5 mutations were linked to juvenile osteoporosis, OPPG, as well as to some high bone mass phenotypes [21]. Mutations in the SOST gene, coding for sclerostin, and inhibitor of the WNT signaling pathway cause high bone mass in two skeletal dysplasias: sclerosteosis and van Buchem disease [22, 23]. In 2013, we reported WNT1 to be a key ligand to WNT signaling in the bone as heterozygous mutations in WNT1 were found to lead to severe early-onset osteoporosis and homozygous mutations, to cause severe OI [24]; a heterozygous missense mutation c.652 T > G (p.C218G) was identified in a large Finnish family with ten affected adults presenting with multiple peripheral and vertebral compression fractures and low BMD, and a homozygous nonsense mutation c. $884 \mathrm{C}>\mathrm{A}$ (p.S295*) was shown to cause severe autosomal recessive OI in two Lao Hmong sisters with multiple fractures, short stature, and long bone deformities, and other groups described similar findings in pediatric and adult patients with WNT1 mutations [25-28] (Table 2, Fig. 1).

Further studies showed equivalent skeletal findings even in children with the heterozygous WNT1 mutation p.C218G [36]; the mutation was observed to affect skeletogenesis and lead to changes in BMD and long bone shape already in early childhood. All mutation-positive children and adolescents presented with low BMD and multiple peripheral, lowenergy fractures. In plain radiographs, the bones were osteopenic in appearance, had growth-arrest lines as a sign of disturbed skeletal development, and the long bones were slightly abnormal in shape (i.e., thin fibulae and overtubulation of the metaphyseal ends of the humera, tibiae, 
Table 2 Reported mutations in WNT1, PLS3, and XYLT2 in association with osteoporosis and other bone mass disorders

\begin{tabular}{|c|c|c|c|c|}
\hline Gene & Location & Mutation & Mono-/biallelic & Reference \\
\hline WNT1 & Exon 2 & c.110 T > C, p.Iso37Thr & Compound heterozygous & {$[29]$} \\
\hline WNT1 & Exon 2 & c. $259 \mathrm{C}>\mathrm{T}, \mathrm{p} . \mathrm{G} \ln 87^{*}$ & Compound heterozygous & {$[26]$} \\
\hline WNT1 & Exon 2 & c.287_300delAGTTCCGGAATCGC, p.Gln96Profs*54 & Homozygous & {$[26]$} \\
\hline WNT1 & Exon 2 & c.287_300del, p.Gln96Profs & Homozygous & {$[27]$} \\
\hline WNT1 & Exon 3 & c.385G > A, p. Ala129Thr & Compound heterozygous & {$[29]$} \\
\hline WNT1 & Exon 3 & c. $428 \mathrm{G}>\mathrm{T}$, p.Cys $143 \mathrm{Phe}$ & Homozygous & {$[27]$} \\
\hline WNT1 & Exon 3 & c.505G > T, P.Gly169Cys & Compound heterozygous & {$[29]$} \\
\hline WNT1 & Exon 3 & c.506dupG, p.Cys170Leufs* & Homozygous & {$[29]$} \\
\hline WNT1 & Exon 3 & c.506G > A, p.Gly169Asp & Compound heterozygous & [29] \\
\hline WNT1 & Exon 3 & c.506dupG, p.Cys170Leufs*6 & Compound heterozygous & {$[26]$} \\
\hline WNT1 & Exon 3 & c.525_536delCTTCGGCCGCCT, p.del176_179 & Heterozygous & {$[30]$} \\
\hline WNT1 & Exon 4 & c. $652 \mathrm{~T}>$ G, p.Cys 218 Gly & Heterozygous & {$[24]$} \\
\hline WNT1 & Exon 4 & c. $884 \mathrm{C}>\mathrm{A}, \mathrm{p}$. Ser $295^{*}$ & Homozygous & {$[24]$} \\
\hline WNT1 & Exon 4 & c.893 T > G, p.Phe298Cys & Homozygous & {$[26]$} \\
\hline WNT1 & Exon 4 & c. 990 C > A, p.Cys $330 *$ & Homozygous & {$[28]$} \\
\hline WNT1 & Exon 4 & c.946_949insAACA, p.Ser317Lysfs & Compound heterozygous & {$[27]$} \\
\hline WNT1 & Exon 4 & c. $1063 \mathrm{G}>\mathrm{T}, \mathrm{p}$. Val355Phe & Compound heterozygous & {$[27]$} \\
\hline PLS3 & Intron 2 & c. $73-24 \mathrm{~T}>\mathrm{A}$ & Heterozygous & {$[31]$} \\
\hline PLS3 & Exon 3 & c.235delT, p.Tyr79Ilefs*6 & Heterozygous & {$[32]$} \\
\hline PLS3 & Exon 7 & c. $748+1 \mathrm{G}>\mathrm{A}$ & Heterozygous & {$[32]$} \\
\hline PLS3 & Exon 8 & c.759_760insAAT, p.Ala253_Leu254insAsn & Heterozygous & {$[32]$} \\
\hline PLS3 & Exon 10 & c994_995delGA, p.Asp332* & Heterozygous & {$[33]$} \\
\hline PLS3 & Exon 13 & c.1433 T > C, p.Leu478Pro & Heterozygous & {$[33]$} \\
\hline PLS3 & Exon 13 & c. $1471 \mathrm{C}>\mathrm{T}, \mathrm{p} . \mathrm{Gln} 491^{*}$ & Heterozygous & {$[32]$} \\
\hline PLS3 & Exon 15 & c.1647delC, p.Ser550Alafs*9 & Heterozygous & {$[32]$} \\
\hline XYLT2 & Exon 2 & p.A174Pfs*35 & Homozygous & {$[34]$} \\
\hline XYLT2 & Exon 3 & p.V232Gfs*54 & Homozygous & {$[34]$} \\
\hline XYLT2 & Exon 8 & p.R563G & Homozygous & {$[35]$} \\
\hline XYLT2 & Exon 9 & p.L605P & Homozygous & {$[35]$} \\
\hline XYLT2 & Exon 10 & p.R730* & Homozygous & {$[35]$} \\
\hline
\end{tabular}

and distal femora) (Fig. 2). Unlike in classical OI, all subjects had normal growth and no apparent dysmorphias or extraskeletal features, and metabolic bone markers, as well as calcium and phosphate parameters in serum and urine, were normal [37]. In recessive forms of WNT1 osteoporosis, the biallelic loss-of-function of WNT1 has more detrimental effects on skeletal growth as severe bone deformities, multiple fractures, and retarded growth are detected soon after birth and can even be postnatally lethal [24-27]. Homozygous WNT1 mutations also lead to a more OI-like phenotype with dentinogenesis imperfecta and blue sclerae, and in various other extra-skeletal abnormalities, such as developmental defects in the central nervous system [24-27].

Questions concerning treatment of WNT1 osteoporosis remain unanswered. While severely affected pediatric patients might benefit from bisphosphonate treatment, care of all patients centers around early preventative measures, including adequate vitamin $\mathrm{D}$ and calcium intake. Our recent clinical trial explored the effects of teriparatide treatment on adults with WNT1 osteoporosis and found an increase in bone formation and BMD in a 24-month treatment period [37]. Anabolic treatment alternatives are presently contraindicated or not available for pediatric patients with WNT1 osteoporosis.

\section{PLS3 Osteoporosis}

Plastin3 (PLS3) functions as a modulator of actin cytoskeleton and forms actin bundles via cross linkage of single actin filaments. While the actin cytoskeleton is known to be important for a wide range of cellular functions, such as endo- and 

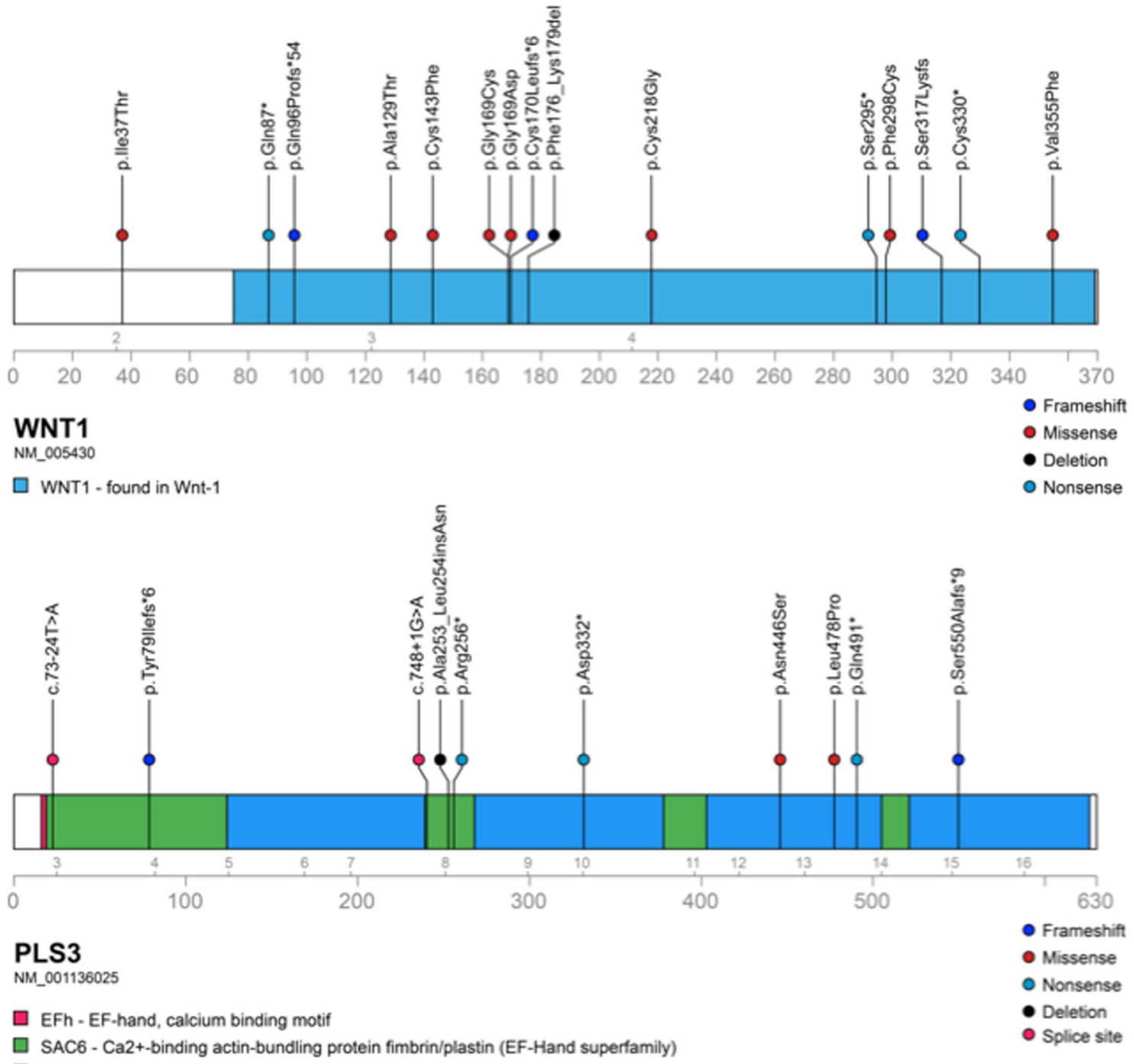

$\square \mathrm{CH}$ - Calponin homology domain

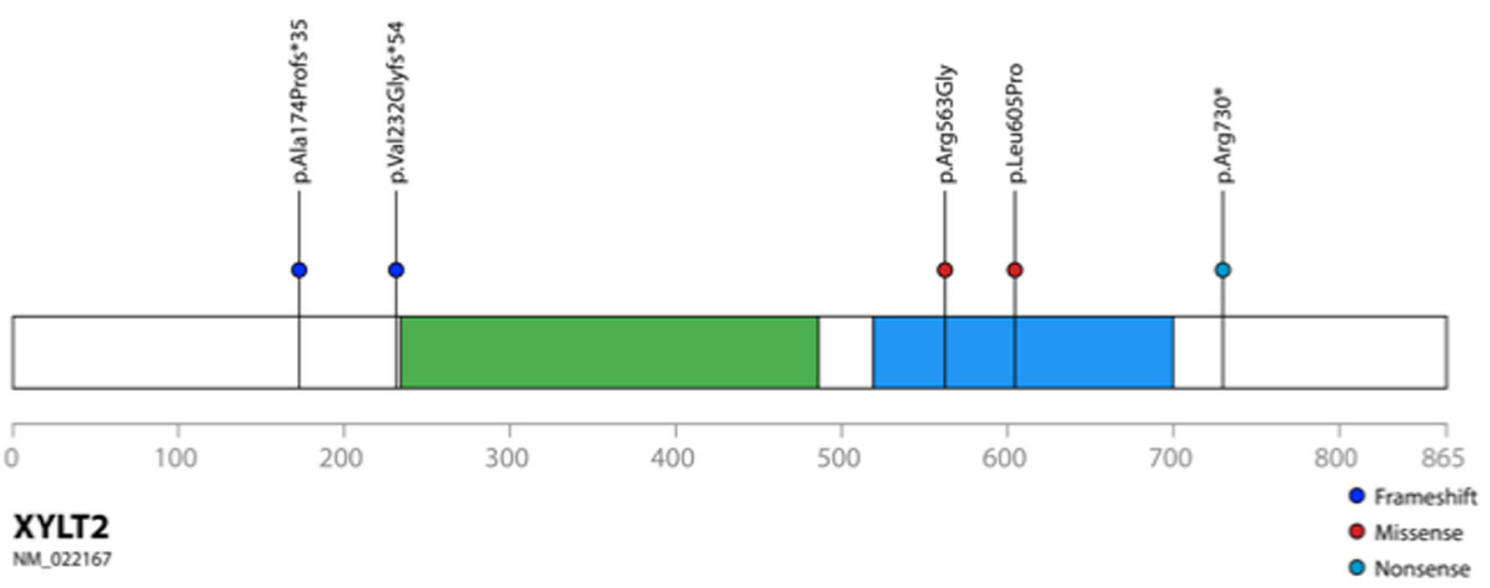

$\square$ Branch-Core-2/1-Branching enzyme

$\square$ Xylo_C-Xylosyltransferase $C$ terminal

Fig. 1 Schematic illustrations of WNT1, PLS3, and XYLT2 proteins. Mutations reported in literature are shown on the proteins. Different types of mutations are labeled in different colors. Numbers above and below the line show exon and amino acid coordinates, respectively based on reference sequences. All schematic illustrations for proteins were generated using protein painter 
Fig. 2 Radiographs of affected patients with heterozygous $W N T 1$ mutation p.C218G or hemizygous $P L S 3$ splice mutation c.73-

$24 \mathrm{~T}>\mathrm{A}$. a Spinal radiograph of an affected 12-year-old male with WNT1 mutation showing one compressed vertebra (arrow). b Spinal radiograph of an affected 62-year-old female with WNT1 mutation showing several severely compressed vertebrae and exaggerated thoracic kyphosis. c Lower extremity radiograph of an affected 30-yearold female with WNT1 mutation showing a thin fibula. d Spinal radiograph of an affected 9-yearold male with $P L S 3$ mutation showing several compressed vertebrae (arrows)

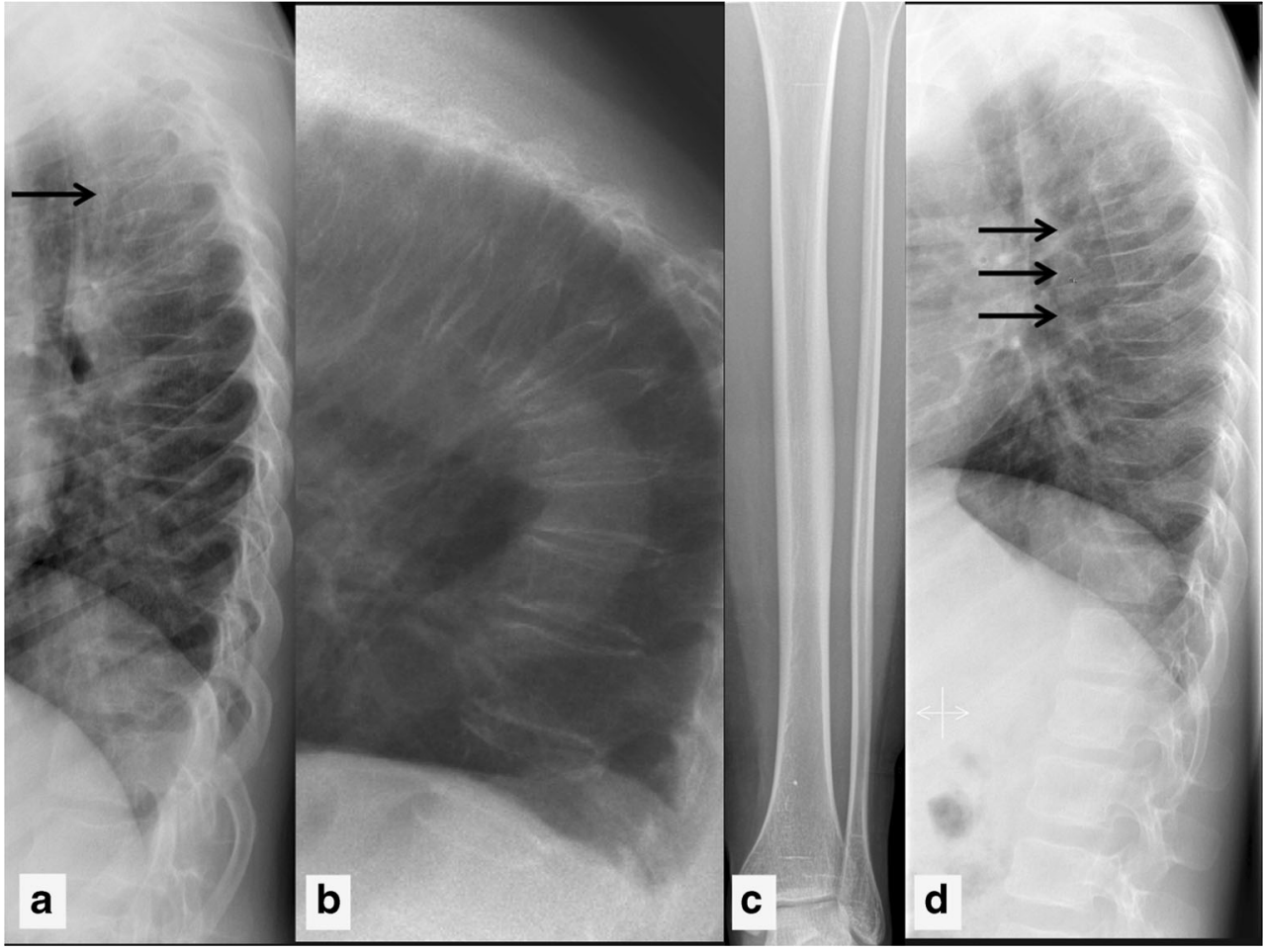

exocytosis, cell migration, and adhesion, its functional significance in specific tissues have been difficult to predict [38, 39]. Studies on spinal muscular atrophy (SMA) have shown evidence that PLS3 participates in neuromuscular synapse formation and maintenance and that overexpression of PLS3 can rescue the axonal defects seen in a mouse model for SMA $[40,41 \bullet \cdot$. In the bone, plastin3 has been proposed to have a role in mineralization and remodeling, possibly even in the osteocytes' mechanosensing apparatus [32, 33].

While the exact mechanisms in bone homeostasis remain incompletely understood, PLS3 has been bestowed a place in the array of monogenic forms of bone mass disorders. In 2013, mutations in the encoding gene PLS3 were first described to cause X-linked early-onset osteoporosis by Van Dijk et al. and since then, other families with PLS3 mutations have been described [31-33]. Due to its X-chromosomal inheritance pattern, males with PLS3 mutations are generally more severely affected. The clinical characteristics are predominated by low BMD, multiple spinal compression fractures, and a range of peripheral fractures (Fig. 2) [31-33].

Of note, although most reported cases of PLS3 osteoporosis have been males, female carriers can also exhibit various skeletal abnormalities. Heterozygous females, with one normally functioning allele, are typically more mildly affected than males but can present with variable clinical traits ranging from subclinical osteopenia to a phenotype resembling that of males with severe osteoporosis, recurrent peripheral fractures, and several vertebral compression fractures in early adulthood [31]. The female carriers also show a more diverse phenotype even within a family. The total number of reported subjects with PLS3 osteoporosis is still very limited (Table 2), and the complete phenotypic spectrum and natural course remain inadequately characterized. Also of importance is the recent finding of another X-linked bone mass disorder; MBTPS2 is the first reported X-linked form of OI and is quite distinct from PLS3 osteoporosis in other OI-related traits, such as blue sclerae, hearing impairment, discolored teeth, and short stature, which are usually seen in these patients [12].

Bisphosphonates have been evaluated in a handful of cases and indicate good treatment response in PLS3 osteoporosis during childhood, but further studies are needed to confirm this $[32,33]$. Our abovementioned clinical study also evaluated teriparatide-response in PLS3 mutation-positive adults and found similar, though slightly diminished, improvement in bone parameters following a 24-month treatment period [37].

\section{XYLT2 Osteoporosis}

Xylosyltransferases, encoded by two homolog genes XYLT1 and XYLT2, catalyze addition of xylose, a sugar, to serine residue of core proteins. This is the first step of biosynthesis of glycosaminoglycans (GAGs), long hydrophilic unbranched polysaccharides that are attached to the core proteins to form proteoglycans (PGs). PGs - especially heparan sulfate and chondroitin sulfate that are abundant on the cell surface and in chondrocyte extracellular matrix (ECM) — create a microenvironment for diverse cellular processes such as signaling, cell adhesion, proliferation, differentiation, and motility. They 
also play a role in chondrocyte maturation and endochondral skeletal development [42].

In 2015, whole exome sequencing in patients with spondylo-ocular syndrome (SOS) (MIM 605822) - a rare syndrome with autosomal recessive inheritance manner, characterized by generalized osteoporosis, spinal compression fractures, platyspondyly, multiple long bone fractures, short stature, cataract, sensorineuronal hearing loss, and many additional organ problems - revealed homozygous frameshift mutations in XYLT2 in three patients from two ethnically different populations [29] (Fig. 1). In 2016, we reported four additional cases with three different homozygous point mutations in XYLT2, expanding the phenotypic and genotypic spectrum of SOS [34]. These patients had slightly milder phenotype compared with that of the previously reported patients, which could be explained by variable pathogenic effect of mutations. Radiological examinations showed osteopenia, kyphosis, severe platyspondyly, increased intervertebral disc, and compression fractures. Lumbar spine BMD Z-scores ranged from -5.9 to -2.5 among the reported patients with molecular diagnosis $[34,35]$. Although the mechanism for positive treatment response is unknown, patients seemed to benefit from pamidronate treatment as BMD and vertebral morphology were observed to improve in follow-up [34, 35].

Unlike WNT1 and PLS3 osteoporosis, XYLT2 osteoporosis is accompanied by severe defects and impairments in many extra-skeletal organs, such as the eyes, ears, heart, kidneys, and central nervous system, which could be explained by indispensable function of xylosyltransferases in these organs. The functional work done by Munns et al. suggested that even though a residual xylotransferase activity due to XYLT1 expression was measured in patient cells, the function of XYLT2expressed xylosyltransferase was not fully compensated by XYLT1-expressed xylosyltransferase, suggesting cell-type and time specific expression pattern and no compensation of enzyme function by the other [35].

\section{Conclusion}

Remarkable advances in uncovering the genetics behind skeletal disorders with bone fragility have been made during the past decade. Although several alleles have been identified, we are still greatly distanced from wholly understanding the hidden puzzles of bone metabolism and discovering all osteoporosis-associated genes. Osteogenesis imperfecta type I serves as the prototype of primary pediatric skeletal dysplasias with skeletal fragility, but future research will probably widen the scope of new forms of primary osteoporosis that do not only concern the extracellular matrix but also some intracellular pathways for bone cell functions. Monogenic and rare forms of osteoporosis help to define and characterize bone metabolism and genetic factors influencing bone health. Thus, genetic reasons should be considered when dealing with fracture prone children, and investigations should target plausible genetic causes in addition to evaluation for secondary causes and lifestyle factors. The underlying genetic cause has important implications for genetic counseling and patient management.

Acknowledgements Our research is financially supported by the Academy of Finland, the Sigrid Jusélius Foundation, the Folkhälsan Research Foundation, the Novo Nordisk Foundation, the Swedish Research Council, the Swedish Childhood Cancer Foundation, the Helsinki University Hospital research funds, and through the regional agreement on medical training and clinical research (ALF) between the Stockholm County Council and the Karolinska Institutet.

\section{Compliance with Ethical Standards}

Conflict of Interest Outi Mäkitie reports payment for lecture and advisory board membership from Alexion and KyoawKirin.

Anders Kämpe, Riikka Mäkitie, and Fulya Taylan declare no conflicts of interest.

Human and Animal Rights and Informed Consent This article does not contain any studies with human or animal subjects performed by any of the authors.

\section{References}

Papers of particular interest, published recently, have been highlighted as:

- Of importance

•- Of major importance

1. Mäkitie O. Causes, mechanisms and management of paediatric osteoporosis. Nat Rev Rheumatol. 2013;9:465-75.

2. NIH Consensus Development Panel on Osteoporosis Prevention, Diagnosis, and Therapy. Osteoporosis prevention, diagnosis, and therapy. JAMA. 2001;285:785-95.

3. Mäkitie O, Doria AS, Henriques F, et al. Radiographic vertebral morphology: a diagnostic tool in pediatric osteoporosis. J Pediatr. 2005;146:395-401.

4. Bishop N, Arundel P, Clark E, International Society of Clinical Densitometry, et al. Fracture prediction and the definition of osteoporosis in children and adolescents: the ISCD 2013 pediatric official positions. J Clin Densitom. 2014;17:275-80.

5. Ralston SH. Genetics of osteoporosis. Ann N Y Acad Sci. 2010;1192:181-9.

6. Zheng HF, Forgetta V, Hsu YH, et al. Whole-genome sequencing identifies EN1 as a determinant of bone density and fracture. Nature. 2015;526:112-7.

7. Guéguen R, Jouanny P, Guillemin F, Kuntz C, Pourel J, Siest G. Segregation analysis and variance components analysis of bone mineral density in healthy families. J Bone Miner Res. 1995;10: 2017-22.

8. Stewart TL, Ralston SH. Role of genetic factors in the pathogenesis of osteoporosis. J Endocrinol. 2000;166:235-45. Review.

9. Bonafe L, Cormier-Daire V, Hall C, et al. Nosology and classification of genetic skeletal disorders: 2015 revision. Am J Med Genet A. 2015;167A:2869-92. This review provides the newly refined classification for different types of osteogenesis imperfecta and other genetic forms of skeletal disorders. 
10. Marini JC, Blissett AR. New genes in bone development: what's new in osteogenesis imperfecta. J Clin Endocrinol Metab. 2013;98: 3095-103.

11. Trejo P, Rauch F. Osteogenesis imperfecta in children and adolescents - new developments in diagnosis and treatment. Osteoporos Int. 2016;27:3427-37.

12. Lindert U, Cabral WA, Ausavarat S, et al. MBTPS2 mutations cause defective regulated intramembrane proteolysis in X-linked osteogenesis imperfecta. Nat Commun. 2016;7:11920.

13. Lindahl K, Åström E, Rubin CJ, et al. Genetic epidemiology, prevalence, and genotype-phenotype correlations in the Swedish population with osteogenesis imperfecta. Eur J Hum Genet. 2015;23:1042-50.

14. Ben Amor IM, Glorieux FH, Rauch F. Genotype-phenotype correlations in autosomal dominant osteogenesis imperfecta. J Osteoporos. 2011;2011:540178

15. Gong Y, Slee RB, Fukai N, et al. LDL receptor-related protein 5 (LRP5) affects bone accrual and eye development. Cell. 2001;107: 513-23.

16. Saarinen A, Saukkonen T, Kivelä T, et al. Low density lipoprotein receptor-related protein 5 (LRP5) mutations and osteoporosis, impaired glucose metabolism and hypercholesterolaemia. Clin Endocrinol. 2010;72:481-8.

17. Balemans W, Ebeling M, Patel N, et al. Increased bone density in sclerosteosis is due to the deficiency of a novel secreted protein (SOST). Hum Mol Genet. 2001;10:537-43.

18. Loots GG, Kneissel M, Keller H, et al. Genomic deletion of a longrange bone enhancer misregulates sclerostin in Van Buchem disease. Genome Res. 2005;15:928-35.

19. Marini JC, Reich A, Smith SM. Osteogenesis imperfecta due to mutations in non-collagenous genes: lessons in the biology of bone formation. Curr Opin Pediatr. 2014;26:500-7.

20.• Baron R, Kneissel M. WNT signaling in bone homeostasis and disease: from human mutations to treatments. Nat Med. 2013;19: 179-92. This article gives a good review of the importance of WNT signaling to bone health.

21. Korvala J, Jüppner H, Mäkitie O, et al. Mutations in LRP5 cause primary osteoporosis without features of OI by reducing Wnt signaling activity. BMC Med Genet. 2012;13:26.

22. Brunkow ME, Gardner JC, Van Ness J, et al. Bone dysplasia sclerosteosis results from loss of the SOST gene product, a novel cysteine knot-containing protein. Am J Hum Genet. 2001;68:577-89.

23. Balemans W, Patel N, Ebeling M, et al. Identification of a $52 \mathrm{~kb}$ deletion downstream of the SOST gene in patients with van Buchem disease. J Med Genet. 2002;39:91-7.

24. Laine CM, Joeng KS, Campeau PM, et al. WNT1 mutations in early-onset osteoporosis and osteogenesis imperfecta. N Engl J Med. 2013;368:1809-16.

25. Keupp K, Beleggia F, Kayserili H, et al. Mutations in WNT1 cause different forms of bone fragility. Am J Hum Genet. 2013;92:565-74.

26. Pyott SM, Tran TT, Leistritz DF, et al. WNT1 mutations in families affected by moderately severe and progressive recessive osteogenesis imperfecta. Am J Hum Genet. 2013;92:590-7.
27. Fahiminiya S, Majewski J, Mort J, Moffatt P, Glorieux FH, Rauch F. Mutations in WNT1 are a cause of osteogenesis imperfecta. J Med Genet. 2013;50:345-8.

28. Faqeih E, Shaheen R, Alkuraya FS. WNT1 mutation with recessive osteogenesis imperfecta and profound neurological phenotype. J Med Genet. 2013;50:491-2.

29. Liu Y, Song L, Ma D, et al. Genotype-phenotype analysis of a rare type of osteogenesis imperfecta in four Chinese families with WNT1 mutations. Clin Chim Acta. 2016;461:172-80.

30. Stephen J, Girisha KM, Dalal A, et al. Mutations in patients with osteogenesis imperfecta from consanguineous Indian families. Eur J Med Genet. 2015;58:21-7.

31. Laine CM, Wessman M, Toiviainen-Salo S, et al. A novel splice mutation in PLS3 causes X-linked early onset low-turnover osteoporosis. J Bone Miner Res. 2015;30:510-8.

32. van Dijk FS, Zillikens MC, Micha D, et al. PLS3 mutations in Xlinked osteoporosis with fractures. N Engl J Med. 2013;369:1529-36.

33. Fahiminiya S, Majewski J, Al-Jallad H, et al. Osteoporosis caused by mutations in PLS3: clinical and bone tissue characteristics. J Bone Miner Res. 2014;29:1805-14.

34. Munns CF, Fahiminiya S, Poudel N, et al. Homozygosity for frameshift mutations in XYLT2 result in a spondylo-ocular syndrome with bone fragility, cataracts, and hearing defects. Am J Hum Genet. 2015;96:971-8.

35. Taylan F, Costantini A, Coles N, et al. Spondyloocular syndrome: novel mutations in XYLT2 gene and expansion of the phenotypic spectrum. J Bone Miner Res. 2016;31:1577-85.

36. Mäkitie RE, Haanpää M, Valta $\mathrm{H}$, et al. Skeletal characteristics of WNT1 osteoporosis in children and young adults. J Bone Miner Res. 2016;31:1734-42.

37. Välimäki VV, Mäkitie $\mathrm{O}$, Pereira R, et al. Teriparatide treatment in patients with WNT1 or PLS3 mutation-related early-onset osteoporosis - a pilot study. J Clin Endocrinol Metab. 2016; doi:10.1210/ jc. 2016-2423.

38. Volkmann N, DeRosier D, Matsudaira P, Hanein D. An atomic model of actin filaments cross-linked by fimbrin and its implications for bundle assembly and function. J Cell Biol. 2001;153:947-56.

39. Lyon AN, Pineda RH, Hao le T, Kudryashova E, Kudryashov DS, Beattie CE. Calcium binding is essential for plastin 3 function in Smndeficient motoneurons. Hum Mol Genet. 2014;23:1990-2004.

40. Oprea GE, Krober S, McWhorter ML, et al. Plastin 3 is a protective modifier of autosomal recessive spinal muscular atrophy. Science. 2008;320:524-7.

41.• Heesen L, Peitz M, Torres-Benito L, et al. Plastin 3 is upregulated in iPSC-derived motoneurons from asymptomatic SMN1-deleted individuals. Cell Mol Life Sci. 2016;73:2089-104. This paper reports the first finding of an $\mathrm{X}$-chromosomal form of $\mathrm{OI}$ and expands our knowledge on the possible inheritance patterns.

42. Taylan F, Mäkitie O. Abnormal proteoglycan synthesis due to gene defects causes skeletal diseases with overlapping phenotypes. Horm Metab Res. 2016;48:745-54. 